\title{
A Proton ENDOR Study of Azurin
}

\author{
Silvia Sottini · Peter Gast • Anneloes Blok • \\ Gerard W. Canters - Davide Cavazzini • \\ Gian Luigi Rossi • Edgar J. J. Groenen
}

Received: 13 May 2009/Revised: 23 June 2009/Published online: 3 November 2009

(C) The Author(s) 2009. This article is published with open access at Springerlink.com

\begin{abstract}
As part of our ongoing project that aims at the optimum characterization of the electronic structure of the blue-copper site of azurin from Pseudomonas aeruginosa, we present the complete hyperfine tensors of the protons bound to the $\mathrm{C} \beta$ atom of the copper-bound cysteine 112. These tensors have been obtained from a $95 \mathrm{GHz}$ pulsed electron-nuclear double resonance study of a single crystal of the protein.
\end{abstract}

\section{Introduction}

Besides the geometric structure of proteins and enzymes, the electronic structure of the active site is an indispensable ingredient in the analysis of the structure-function relationship of such biosystems. Whether the function involves electron transfer, bond breaking, and/or bond formation, in all cases rearrangement of the electron distribution is under discussion. For active sites carrying one or more transition metal ions in a paramagnetic oxidation state, the most informative method to study the electronic structure is electron paramagnetic resonance (EPR) spectroscopy.

Here, we consider the type 1 or blue-copper site, which is involved in electron transfer in a variety of proteins and enzymes. A conserved property of the type 1 coordination is the ligation of copper by three strong equatorial ligands, the sulfur

S. Sottini · P. Gast · E. J. J. Groenen $(\bowtie)$

Huygens Laboratory, Leiden University, P.O. Box 9504, Niels Bohrweg 2,

2333 CA Leiden, The Netherlands

e-mail: mat@molphys.leidenuniv.nl

A. Blok · G. W. Canters

Gorlaeus Laboratories, Metallo Protein Group, Leiden University, Einsteinweg 55,

2333 CC Leiden, The Netherlands

D. Cavazzini · G. L. Rossi

Department of Biochemistry and Molecular Biology, University of Parma,

Parco Area delle Scienze 23 A, 43100 Parma, Italy 
atom $\mathrm{S} \gamma$ of a cysteine residue and the nitrogen atoms $\mathrm{N} \delta$ of two histidines. The copper is about in the plane of these sulfur and nitrogen atoms, and a fourth, axial ligand is present, commonly the sulfur of a methionine. In particular, two proteins, plastocyanin and azurin, have extensively been studied as their copper sites are characteristic for this coordination. Plastocyanin, because it was the first bluecopper protein that was crystallized, and whose structure was derived from X-ray diffraction studies [1]. Second, azurin (Fig. 1), because it was the first blue-copper protein that was genetically modified. Its structure was available soon after that of plastocyanin [2, 3]. Azurin and mutants thereof have extensively been used to investigate the properties of the blue-copper site including its electronic structure [4].

During the last decades, developments have taken place in EPR instrumentation, which have significantly broadened the applicability of this technique to biosystems. These developments concern the use of pulsed microwave excitation [5] and the development of spectrometers that operate at microwave frequencies significantly higher than $9 \mathrm{GHz}$ and correspondingly high magnetic fields [6]. Apart from resolution enhancement, these spectrometers show an increased sensitivity for small samples. In 1994, we realized that this could enable the study of protein crystals, for which a submillimeter size is common. Indeed, since then we have investigated single crystals of azurin by electron spin echo (ESE)-detected EPR at $95 \mathrm{GHz}$, which resulted in complete tensorial information on $g$ [7] and the hyperfine and quadrupole interaction of the copper-coordinated and remote nitrogens of the histidine residues [8,9]. The nitrogen hyperfine tensors nicely reveal the (asymmetric) delocalization of the unpaired electron over the histidine residues, but the delocalization over the cysteine residue remained undetermined. This is unsatisfactory because the strong covalent character of the copper-sulfur bond is considered the decisive property of the blue-copper site. Quantification of the extent of the delocalization of the electronic wave function over the copper-bound sulfur is, in principle, possible by pulsed ${ }^{33} \mathrm{~S}$ electron-nuclear double resonance (ENDOR).

Fig. 1 The copper site of azurin. The copper is coordinated by three strong equatorial ligands $\left(\mathrm{N}_{\delta}\right.$ of histidine 46 and 117 and $\mathrm{S}_{\gamma}$ of cysteine 112). The sulfur of a methionine and the oxygen of a glycine provide two weak axial ligands. The Cys- $\beta \mathrm{Hs}$ are also shown

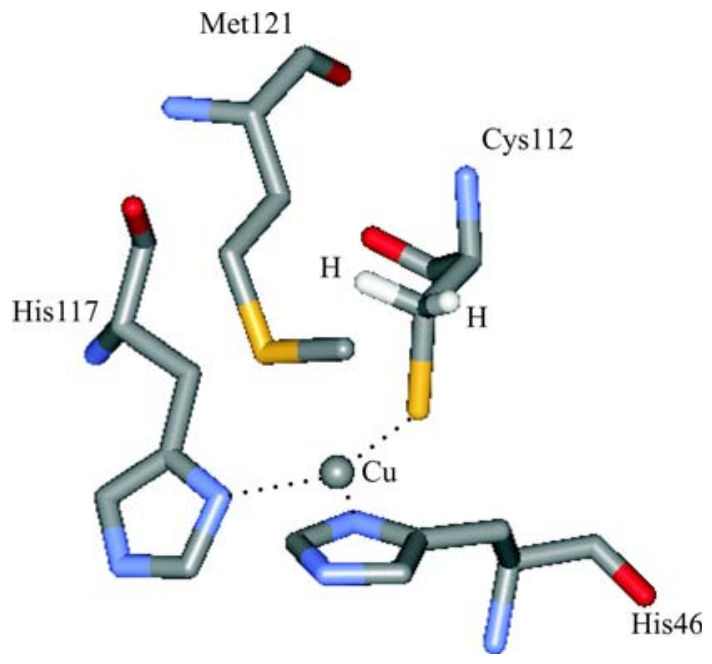


This would require isotope enrichment of the protein, as the natural abundance of ${ }^{33} \mathrm{~S}$ is only $0.75 \%$. Instead, we study the hyperfine interaction of the unpaired electron with the nuclear spin of the protons bound to the $\mathrm{C} \beta$ atom of cysteine (Cys$\beta \mathrm{Hs}$ ), which provides a measure of the copper-cysteine ligation.

In an attempt to determine the complete hyperfine tensors of the Cys- $\beta \mathrm{Hs}$, we recently studied an isotopomer of azurin in which the two Cys- $\beta \mathrm{Hs}$ of the cysteine 112 ligand were replaced by deuterium [10]. Because of the quadrupole moment of deuterium, this study, in principle, provided information on the orientation of the $\mathrm{C}-$ $\mathrm{D}(\mathrm{H})$ bonds in the copper site, as well. The analysis of these experiments turned out to be difficult and only partly successful, which led us to the present complementary study of the wild-type azurin.

Our efforts to push on the characterization of the copper site of azurin by EPR are also driven by the fact that the calculation of EPR parameters has become a critical test of the quantum-chemical description of the blue-copper site. Various ab initio and density functional theory (DFT) approaches have shown that a proper description of the electronic structure of this copper coordination is far from trivial, but progress has been made during the last years [11-13]. The availability of hyperfine tensors of ligand nuclei, as an experimental measure of the extent of the electronic wave function, allows a critical test of the calculated wave function of the ground state. In this sense, the hyperfine tensors of the Cys- $\beta$ Hs would be most valuable data.

In this paper, we report on a pulsed ENDOR study of a single crystal of the bluecopper protein azurin from Pseudomonas aeruginosa from which we derive the complete hyperfine tensors of the Cys- $\beta$ Hs.

\section{Materials and Methods}

Azurin from P. aeruginosa was purified as described elsewhere [14]. Crystals of the oxidized protein were grown by vapor diffusion, as reported previously [3]. Typical dimensions of the crystals studied were $0.5 \times 0.3 \times 0.3 \mathrm{~mm}$. The space group of the crystals is $P 2{ }_{1} 2_{1} 2_{1}$ and the unit cell contains 16 molecules, with 4 molecules per asymmetric unit. The single crystal was mounted in a quartz tube with the inner and outer diameters of 0.6 and $0.84 \mathrm{~mm}$, respectively. The tube ends were sealed with wax. The sample tube and the magnet could be rotated around independent axes, thus enabling experiments at every possible orientation of the magnetic field with respect to the crystal, without remounting the crystal.

The EPR and ENDOR experiments were performed at $2 \mathrm{~K}$, using a homebuilt $95 \mathrm{GHz}$ pulsed EPR spectrometer [15]. A Mims-type three-pulse ENDOR sequence was used and the length of the microwave pulses was $260 \mathrm{~ns}$. The time between the first and the second microwave pulse was $420 \mathrm{~ns}$, the time between the second and the third microwave pulse was optimized for the different orientations and varied between 35 and $60 \mu \mathrm{s}$. The repetition rate varied between 0.1 and $0.5 \mathrm{kHz}$.

An EPR study was performed on the azurin, crystal to determine the orientation in the laboratory frame of the $g$-tensor principal axes systems $x, y, z$ of the 16 molecules in the unit cell. Subsequently, one molecule was chosen for the proton ENDOR measurements. In addition, the nitrogen ENDOR spectrum of the remote 
nitrogens of the histidines was measured on the same crystal at an orientation of $150^{\circ}$ from $x$ in the $x y$ plane of the $g$-tensor principal axes system of this molecule. As this spectrum is known from previous experiments [9], this measurement allows us to consistently couple the present data to the previous ones. In this way, we are able to translate the observed directions of the principal axes of the hyperfine tensors into directions in the copper site.

\section{Results}

For many orientations of the magnetic field with respect to a single crystal of azurin, ESE-detected EPR spectra have been acquired. The orientation in the laboratory frame of the $g$-tensor principal axes was obtained for all the 16 azurin molecules in the unit cell. One molecule was then selected and the proton ENDOR experiments were performed for many orientations of the magnetic field with respect to the crystal, adjusting the field strength to remain in resonance with the EPR transition of that molecule. Orientations were chosen in four planes, three of which concerned the principal planes of the $g$-tensor. In Fig. 2, a spectrum acquired with the magnetic field parallel to $x$ and at field strength of $3.326 \mathrm{~T}$ is shown. Two broad ENDOR transitions are observed at about $10 \mathrm{MHz}$ from the hydrogen Zeeman frequency $v_{\mathrm{Z}}$ $(141.589 \mathrm{MHz})$, which originate from the Cys- $\beta \mathrm{Hs}$ of the copper bound cysteine. From one hydrogen atom $(I=1 / 2)$, we expect to see two ENDOR transitions, symmetrically displaced with respect to the nuclear Zeeman frequency.

Fig. 2 The proton ENDOR spectrum of an azurin single crystal for the magnetic field parallel to the principal $x$ axis $(B=3.3226 \mathrm{~T})$ of one of the molecules in the unit cell. Only the region around the hydrogen nuclear Zeeman frequency, which is indicated by the arrow, is shown

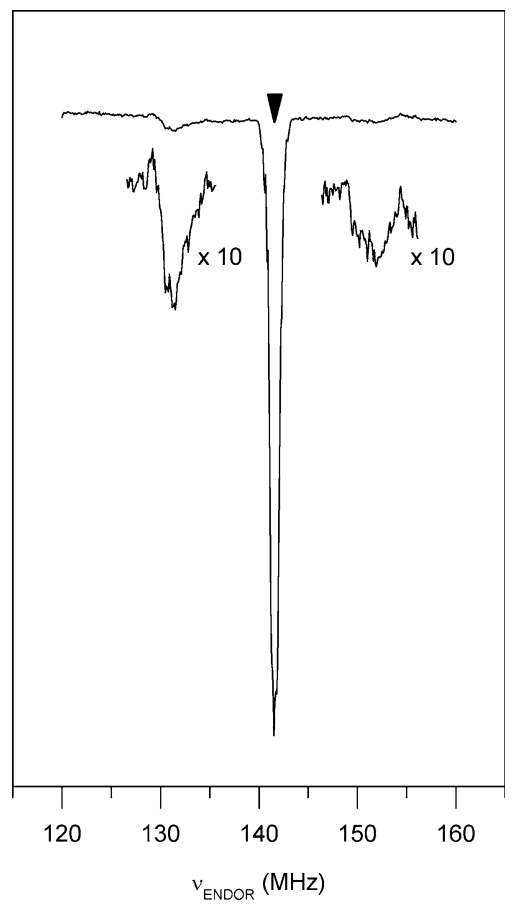


Figure 3 shows ENDOR spectra acquired for various orientations of the magnetic field in the principal planes of the $g$-tensor. Only one of the two ENDOR transitions around the nuclear Zeeman frequency is presented. For each orientation, a broad ENDOR signal is observed, with line width varying between 2 and $3.5 \mathrm{MHz}$. The signal derives from the superposition of two transitions owing to the two hydrogen nuclei, which results in band shapes with varying asymmetry. In the analysis, the ENDOR signals were approximated by two Gaussian functions of equal line width. In this way, the frequencies of the ENDOR transitions of the two hydrogen nuclei were extracted for each orientation of the magnetic field with respect to the crystal. These frequencies are represented in Fig. 4 and were analyzed according to the following equation:

$$
h v_{\mathrm{ENDOR}}^{ \pm}=\left\{\sum_{\beta}\left(-g_{\mathrm{H}} \mu_{\mathrm{N}} B \ell_{\beta} \pm \frac{1}{2} \sum_{\alpha} \ell_{\alpha} g_{\alpha} A_{\alpha \beta} / g\right)^{2}\right\}^{\frac{1}{2}}
$$

Here $\alpha, \beta=x, y, z$ and $x, y, z$ represent the principal axes system of the $g$-tensor. The unit vector $\ell$ represents the direction of the magnetic field $\vec{B}$ in $x, y, z$. The $g_{\alpha}$ values represent the principal values of the $g$-matrix, and

Fig. 3 The proton ENDOR spectra of an azurin single crystal for various orientations of the magnetic field in the $x y, x z$ and $y z$ planes of the $g$-tensor. The $x$ direction corresponds to $0^{\circ}$ in the $x y$ and $x z$ planes; the $y$ direction corresponds to $0^{\circ}$ for the $y z$ plane. The ENDOR shift is defined as $v_{Z}-v_{\text {ENDOR }}$
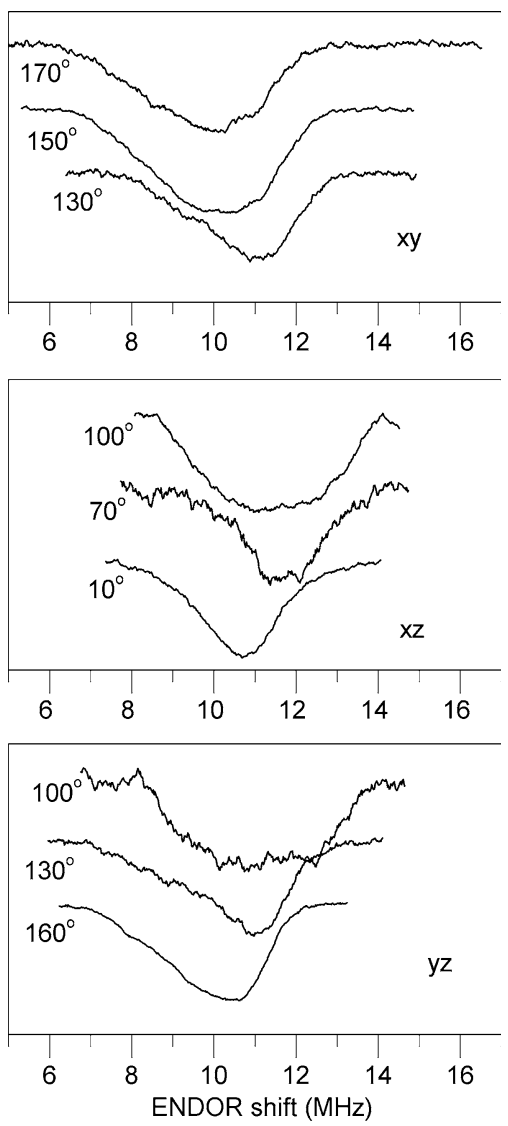


$$
g=\left\{\sum_{\alpha} \ell_{\alpha}^{2} g_{\alpha}^{2}\right\}^{\frac{1}{2}} .
$$

The lines in Fig. 4 represent the fit of the ENDOR frequencies based on Eq. (1) and the resulting hyperfine tensors are summarized in Table 1. The fit nicely describes most of the ENDOR frequencies, but for a few orientations, the experimental data deviate significantly, up to $1 \mathrm{MHz}$, from the calculated frequencies. This deviation, which is still within the line width of the ENDOR

Fig. 4 The proton ENDOR resonances as a function of the orientation of the magnetic field in the following planes: $x y$ (a), $y z$ (b), $x z$ (c), and the plane containing $z$ and crossing the $x y$ plane at $130^{\circ}$ from $x(\mathbf{d})$. The dots (squares for $\mathrm{H}_{1}$ and circles for $\mathrm{H}_{2}$ ) represent the data points, the lines (solid for $\mathrm{H}_{1}$ and dotted for $\mathrm{H}_{2}$ ) are the fits based on Eq. (1). The ENDOR shift is defined as $v_{Z}-v_{\text {ENDOR }}$
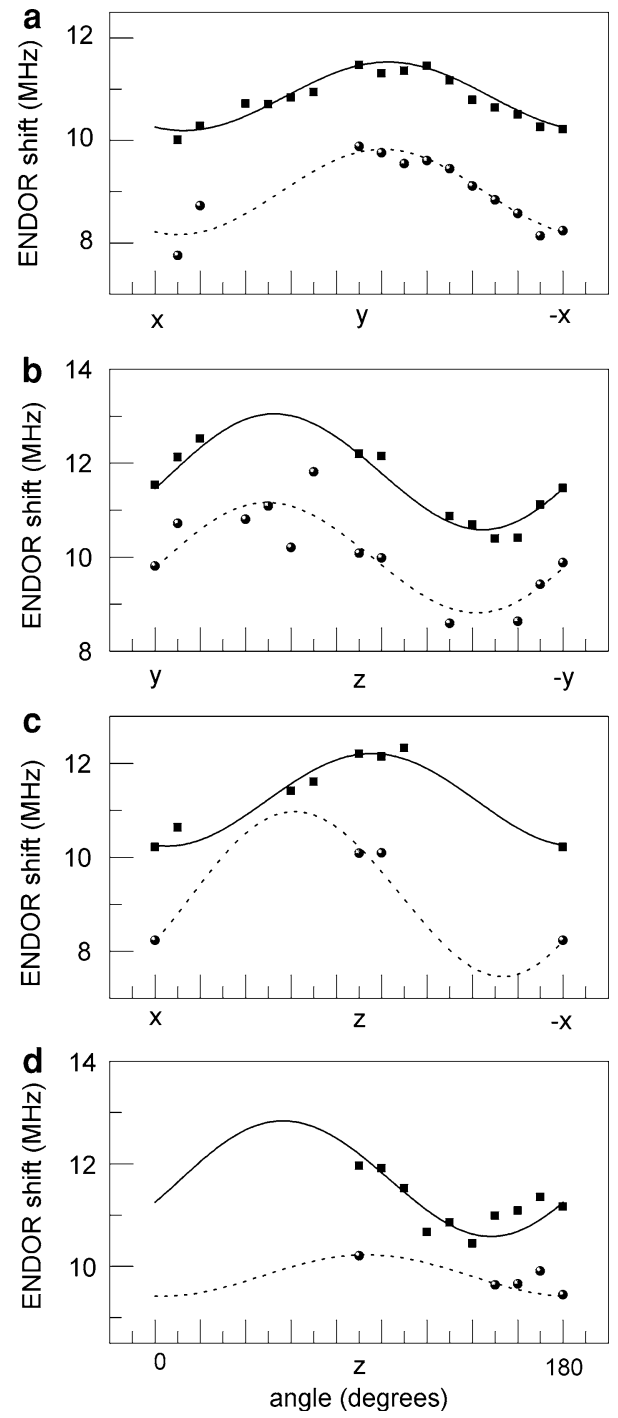
Table 1 Hyperfine tensors of the two hydrogen nuclei as obtained from the fit of the ENDOR frequencies using Eq. (1)

\begin{tabular}{|c|c|c|c|c|c|c|c|c|}
\hline & $A_{\text {iso }} / h$ & $A_{x^{\prime} x^{\prime}} / h$ & $A_{y^{\prime} y^{\prime}} / h$ & $A_{z^{\prime} z^{\prime}} / h$ & & $x^{\prime}$ & $y^{\prime}$ & $z^{\prime}$ \\
\hline \multirow[t]{3}{*}{$\mathrm{H}_{1}$} & $22.6(0.3)$ & $-2.2(0.6)$ & $-1.3(0.6)$ & $3.6(0.6)$ & $x$ & 0.955 & -0.272 & -0.114 \\
\hline & & & & & $y$ & 0.285 & 0.752 & 0.594 \\
\hline & & & & & $z$ & -0.080 & -0.600 & 0.796 \\
\hline \multirow[t]{3}{*}{$\mathrm{H}_{2}$} & $18.8(0.4)$ & $-4.4(0.8)$ & $0.25(0.8)$ & $4.15(0.8)$ & $x$ & 0.805 & 0.504 & 0.314 \\
\hline & & & & & $y$ & 0.316 & -0.811 & 0.492 \\
\hline & & & & & $z$ & -0.503 & 0.297 & 0.812 \\
\hline
\end{tabular}

The hyperfine values are expressed in MHz. The tensors are decomposed in their isotropic and anisotropic contribution. The values in brackets represent the errors in the tensor elements. The direction cosines represent the directions of the principal axes systems of the proton hyperfine tensors $\left(x^{\prime}, y^{\prime}, z^{\prime}\right)$ in the principal axes system $(x, y, z)$ of the $g$-tensor. We have taken $A_{\text {iso }}$ positive

signal, results from the fact that for these orientations a reliable decomposition of the ENDOR band into two Gaussians was not possible.

\section{Discussion}

Proton ENDOR spectra have been obtained for many orientations of the magnetic field with respect to one molecule in the unit cell of a single crystal of wild-type azurin from $P$. aeruginosa. The ENDOR shifts observed for the magnetic field applied along the principal $y$ and $z$ axes of the $g$ tensor correspond to shifts previously measured for frozen solutions by Epel et al. [16] at $95 \mathrm{GHz}$ and Werst et al. [17] at $35 \mathrm{GHz}$. From the present experiments on the single crystal, the complete hyperfine tensors of the Cys- $\beta$ Hs have been derived (Table 1).

The two $\mathrm{C} \beta$ hydrogens of cysteine 112 have different isotropic hyperfine interactions, which amount to 22.6 and $18.8 \mathrm{MHz}$. These values yield estimates of the spin densities on the hydrogens of 1.6 and $1.3 \%$, as unit spin density in a hydrogen $1 s$ orbital corresponds to an isotropic hyperfine interaction of $1420 \mathrm{MHz}$.

In a previous pulsed ENDOR study, we investigated an isotopomer of azurin in which the $\mathrm{C} \beta$ hydrogens of cysteine 112 were substituted by deuterium [10]. This substitution introduces nuclei with a quadrupole moment. The idea was that the analysis of the quadrupole interaction would provide the orientation of the $C-D(H)$ bonds in the copper site and thereby support the interpretation of the hyperfine tensors. The comparison of the ENDOR signals for the wild type and deuterated azurin reveals no noticeable differences. This is illustrated in Fig. 5 for the ENDOR spectra that correspond to the orientations of the magnetic field along the principal axes of the $g$-tensor. After rescaling $\left(g_{\mathrm{H}} / g_{\mathrm{D}}=6.5144\right)$, the ENDOR bands overlap and specific contributions of the quadrupole interaction for the deuterated azurin cannot be discerned. All ENDOR bands are broad, which seems to derive from an inhomogeneous distribution of hyperfine interactions.

The anisotropy of the hyperfine interaction of the Cys- $\beta \mathrm{Hs}$ amounts to at most $23 \%$. It is this anisotropic part of the hyperfine tensors that contains the information 
Fig. 5 Comparison of the proton ENDOR spectra of wildtype azurin (solid lines) for directions of the magnetic field parallel to $x, y$ and $z$ with the corresponding deuterium ENDOR spectra of deuterated azurin (dotted lines) from Ref. [10]. The spectra of the wildtype azurin have been rescaled with the factor $g_{\mathrm{H}} / g_{\mathrm{D}}=6.5144$
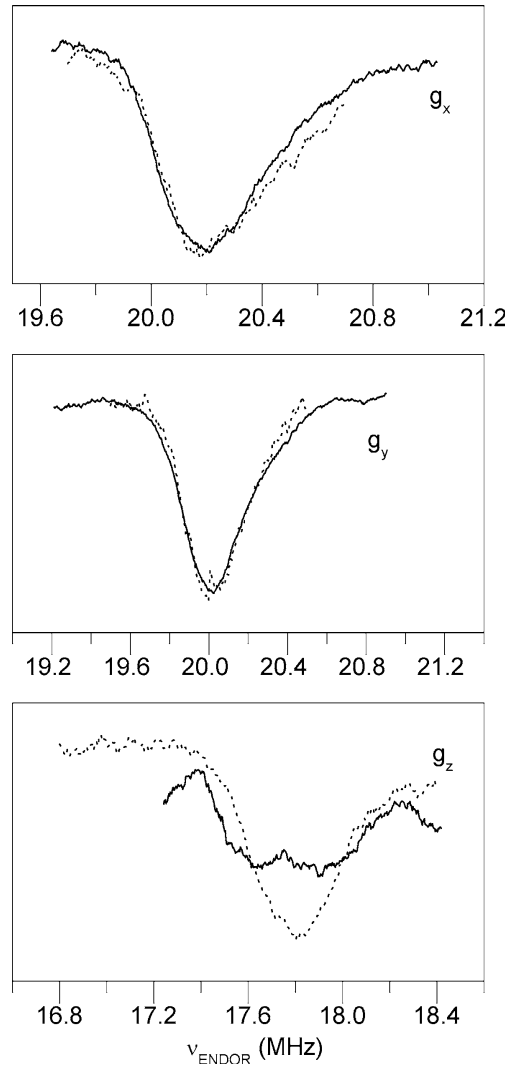

as regards the extent of the electron-spin distribution over the copper atom and the cysteine ligand. The anisotropic hyperfine interaction is proportional to the spin density and inversely proportional to the third power of the distance between the electron-spin density and the nucleus. The spin density at the $\mathrm{C} \beta$ is small but near, while the spin densities at the $\mathrm{S} \gamma$ and $\mathrm{Cu}$ are much larger but more remote. Only the contribution of spin density on the histidine residues to the anisotropic proton hyperfine is negligible because of their large distance to the hydrogens. To reproduce the anisotropy therefore requires a wave function that properly describes the spin-density distribution between the copper and sulphur atoms. For this reason, the present data are most useful when it comes to judge the quality of a quantumchemical description of the blue-copper site.

From a quantum-chemical point of view, the quantitative description of the bluecopper site has been found difficult. Recently, significant progress was reported, which shows that inclusion of e.g., the backbone amide of asparagine 47 that forms a hydrogen bond with the cysteine sulfur and of the protein matrix is a prerequisite of an adequate description $[11,13]$. However, as yet no wave function has been demonstrated to reproduce both the optical absorption spectrum, the $g$-tensor and the (super)hyperfine tensors. The full set of EPR parameters known for the copper site of azurin makes this protein the ideal test system for any quantum-chemical 
calculation, be it based on ab initio theory or DFT. To reproduce the anisotropy of the proton hyperfine tensors that result from the present study really is the critical test.

Acknowledgment The research was supported with financial aid by The Netherlands Organization for Scientific Research (NWO), Department of Chemical Sciences (CW).

Open Access This article is distributed under the terms of the Creative Commons Attribution Noncommercial License which permits any noncommercial use, distribution, and reproduction in any medium, provided the original author(s) and source are credited.

\section{References}

1. P.M. Colman, H.C. Freeman, J.M. Guss, M. Murata, K.A. Norris, J.A.M. Ramshaw, M.P. Venkatappa, Nature 272, 319 (1978)

2. E.T. Adman, L.H. Jensen, Israel J. Chem. 21, 8 (1981)

3. H. Nar, A. Messerschmidt, R. Huber, M. van der Kamp, G.W. Canters, J. Mol. Biol. 221, 765 (1991)

4. U. Kolczak, C. Dennison, A. Messerschmidt, G.W. Canters, Azurin and Azurin Mutants, in Handbook of Metalloproteins, ed. by K. Wieghardt, R. Huber, T.L. Poulos, A. Messerschmidt (Wiley, Chichester, 2001), p. 1170

5. A. Schweiger, G. Jeschke, Principles of Pulse Electron Paramagnetic Resonance (Oxford University Press, Oxford, 2001)

6. O. Grinberg, L.J. Berliner (ed.), Very High Frequency ESR/EPR. Biological Magnetic Resonance, vol. 22 (Kluwer, New York, 2004)

7. J.W.A. Coremans, O.G. Poluektov, E.J.J. Groenen, G.W. Canters, G.W. Nar, A. Messerschmidt, J. Am. Chem. Soc. 116, 3097 (1994)

8. J.W.A. Coremans, O.G. Poluektov, E.J.J. Groenen, G.W. Canters, G.W. Nar, A. Messerschmidt, J. Am. Chem. Soc. 119, 4726 (1997)

9. J.W.A. Coremans, O.G. Poluektov, E.J.J. Groenen, G.W. Canters, G.W. Nar, A. Messerschmidt, J. Am. Chem. Soc. 118, 12141 (1996)

10. M. Fittipaldi, G.C.M. Warmerdam, E.C. de Waal, G.W. Canters, D. Cavazzini, G.L. Rossi, M. Huber, E.J.J. Groenen, Chem. Phys. Chem. 7, 1286 (2006)

11. S. Sinnecker, F. Neese, J. Comput. Chem. 27, 1463 (2006)

12. C. Remenyi, R. Reviakine, M. Kaupp, J. Phys. Chem. B 111, 8290 (2007)

13. S. Vancoillie, K. Pierloot, J. Phys. Chem. A 112, 4011 (2008)

14. M. van de Kamp, F.C. Hali, N. Rosato, A. Finazzi Agro, G.W. Canters, Biochim. Biophys. Acta 1019, 283 (1990)

15. J.A.J.M. Disselhorst, H. van der Meer, O.G. Poluektov, J. Schmidt, J. Magn. Reson. Ser. A 115, 183 (1995)

16. B. Epel, C.S. Slutter, F. Neese, P.M.H. Kroneck, W.G. Zumft, I. Pecht, O. Farver, Y. Lu, D. Goldfarb, J. Am. Chem. Soc 124, 8152 (2002)

17. M.M. Werst, C.E. Davoust, B.M. Hoffman, J. Am. Chem. Soc. 113, 1533 (1991) 